\title{
One Inch CCD Cameras for Neutron and X-ray Imaging
}

\author{
Alan Hewat \\ NeutronOptics and Institut Laue-Langevin, Grenoble, France \\ alan.hewat@neutronoptics.com
}

\section{Keywords: CCD Cameras, Neutron Imaging, X-ray Imaging, Laue Diffraction}

\begin{abstract}
Most normal applications of neutron and x-ray imaging and diffraction can be satisfied with inexpensive commercial CCD cameras. Here we have chosen the largest CCD made by Sony, the 1-inch ICX694ALG, and compared it with much more expensive CCD and CMOS cameras offered by specialized companies such as Andor and PCO. We give examples of the use of this CCD for large area (250x200mm) imaging with optical resolution of $90 \mathrm{um}$, for very high resolution 1:1 macro imaging, where the optical resolution and area is equal to the CCD chip, and for backscattered Laue diffraction where high efficiency is required.
\end{abstract}

\section{Introduction}

Until recently, neutron imaging has been the domain of a few big laboratories in Europe and the USA using expensive equipment. However, neutron imaging is one of the applications that can be conducted on low-flux neutron sources in small laboratories, and indeed it has been a priority for the IAEA in encouraging peaceful uses of nuclear techniques in developing countries. Sufficient neutron intensity can be provided by a 1MW TRIGA reactor, of which there are many in US Universities and in the national laboratories of smaller countries. The TRIGA reactor is very safe, uses relatively low technology, and can be switched on and off as required. Neutron generators using "table top" Deuterium-Deuterium (D-D) or Deuterium-Tritium (D-T) generators are being developed to provide alternative low-flux sources suitable for neutron imaging. A lowcost camera suitable for such low-flux sources, can be used with these new neutron and x-ray generators. Our objective is to satisfy the demand for the wider application of neutron and x-ray imaging. But there is no point in doing "second class" science, so we will show that these low cost cameras can in many cases compete with the more expensive equipment in big laboratories.

\section{The origins of Neutron Cameras, and the Advantages of Simplification}

Neutron cameras are almost as old as neutron diffraction itself, and are simply a variation of the even older photographic techniques used from the discovery of x-rays. Indeed neutron cameras are just X-ray cameras with a component (usually LiF) to convert neutrons into ionising particles and x-rays, which are then converted into light using an x-ray scintillator (usually ZnS). A neutron Polaroid film camera was used with a scintillator from the beginning of ILL in the early 1970 's, and even earlier elsewhere.

The idea of using a video camera instead of film goes back to Arndt \& Ambrose (1968) and was proposed for neutron diffraction by Arndt \& Gilmore (1975), with experiments in the 1970's at ILL Grenoble [1]. CCD cameras were used for the ILL NEUTROGRAPH in 2002 [2]. ILL was the world's highest flux neutron source, with intensities of more than an order of magnitude greater than other facilities, but the NEUTROGRAPH had relatively low resolution and dynamic range. ILL eventually stopped neutron imaging but FRM-2 and HMI Germany with PSI Switzerland in particular, continued to develop the technique. Only recently has ILL built a new world class neutron imaging station, on a high resolution and high flux cold guide [3]. 
ILL did however, continue to develop CCD cameras for sample alignment and diffraction. In 2005, a simple neutron CCD camera [4] was designed to replace the Polaroid neutron camera on most ILL neutron instruments. CCD detectors were also developed for neutron Laue diffraction $[5,6,7]$, and proved a great success, partly replacing neutron image plate detectors.

Our current CCD cameras have a number of technical advantages:

- We use high volume commercial CCD units, which are inexpensive to repair \& replace.

- Neutron scintillators, front-surfaced mirrors and lenses are identical to those used in leading neutron imaging laboratories.

- We use fixed geometry, with a variable Field-of-View (FOV) in our large cameras obtained by simply exchanging the scintillator front end to change the optical path length.

- This fixed geometry requires no translation components that can fail or malfunction.

- More importantly, fixed geometry means that the CCD unit can attached to the exterior of the camera box, can be easily shielded, and can use simple air and Peltier cooling.

- If the CCD unit was on a motorised rail, it would have to be inside the box or external bellows, and the camera would be more complicated, perhaps requiring water cooling.

- Ordinary $12 \mathrm{~V}$ power supplies are used, together with standard USB-2 cables with amplified $10 \mathrm{~m}$ extensions up to $30 \mathrm{~m}$ total.

- A wide choice of third party software is available for free, as well as an SDK.

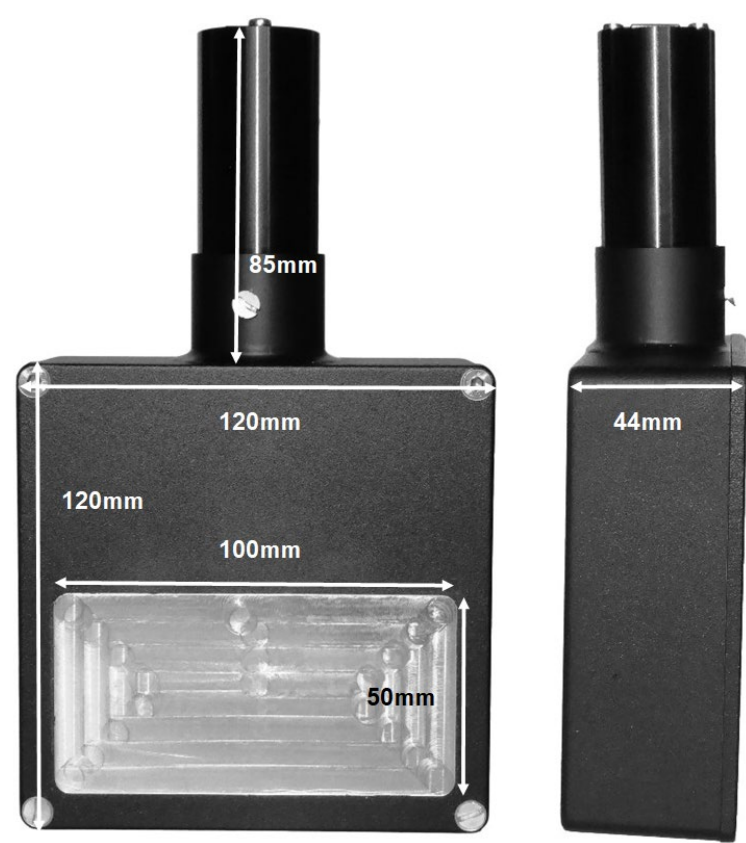

Fig 1. Simple neutron alignment camera

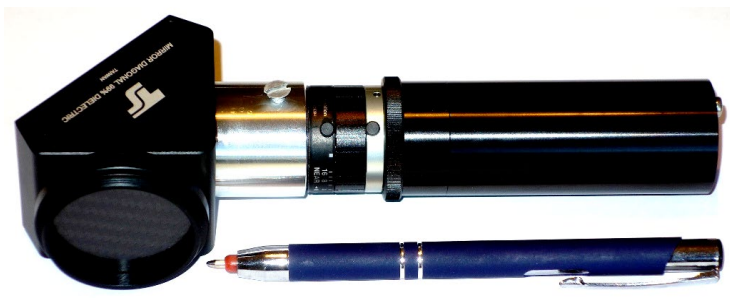

Fig 2. mini-iCam x-ray alignment camera

\section{Simple Neutron and X-ray Beam Alignment camera}

Our current slim camera (fig.1) is designed for checking sample centering and beam homogeneity. It is only $44 \mathrm{~mm}$ thick for a sensitive area of $100 \times 50 \mathrm{~mm}$ using a $1 / 2$ " or $2 / 3$ " Sony CCD, so can fit into the small space between the sample environment and the beam stop. A single 10-20m amplified USB cable is used for power, control and image acquisition.

These simple cameras can be made as large as 200x150mm for guide tube beam homogeneity measurements. With such a large CCD they are very efficient, and weak $10^{4} \mathrm{n} \cdot \mathrm{cm}^{-2} \cdot \mathrm{s}^{-1}$ neutron beams require only a few seconds exposure. Thin but strong carbon fibre windows with appropriate scintillators are used for the similar x-ray cameras.

Our smallest mini-iCam (fig.2) neutron or x-ray camera is only $\sim 190 \mathrm{~mm}$ long and has 580 or 1040 pixels over an area of $30 \mathrm{~mm}$ diameter, so with its efficient f/1.0 lens it is also very bright. The scintillator and carbon fibre window can be exchanged, depending on whether x-rays or neutrons are to be imaged 


\section{High Resolution Neutron \& X-ray Imaging Cameras using a 1-inch CCD}

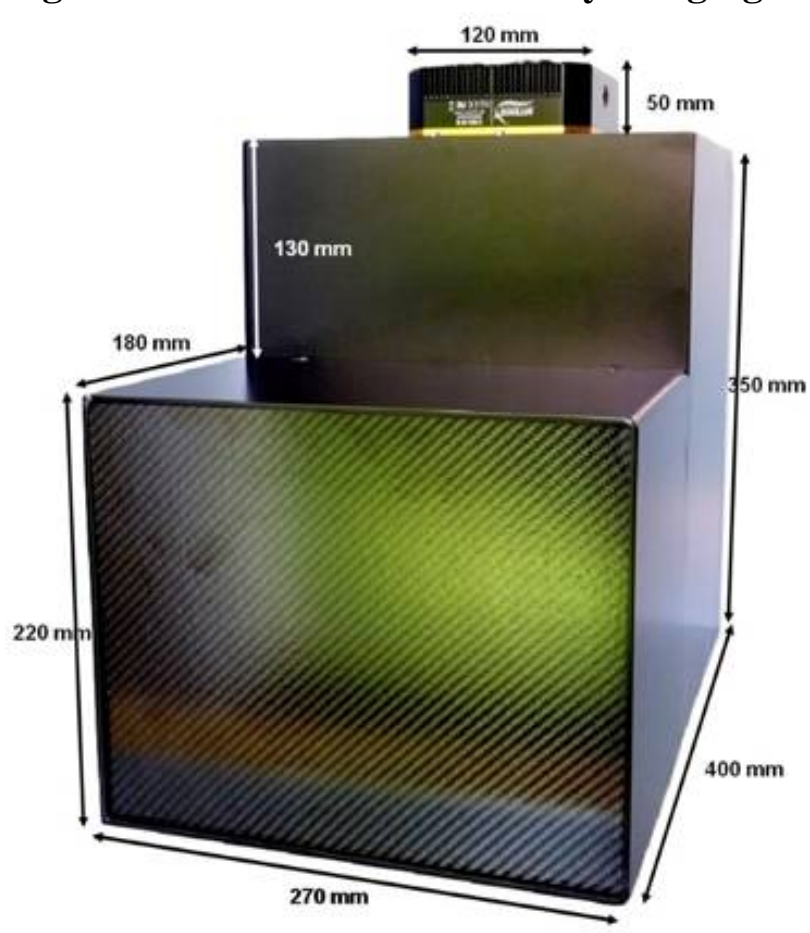

Our imaging camera (fig.3) compares well with more expensive cameras, with low noise, high resolution and fast readout. The scintillator holder on the front section can be changed in-situ to select different neutron and $\mathrm{x}$-ray scintillators (figs.4). For tomography, both software and hardware triggering (via a GPIO socket) is provided.

- Sensor: 1" Sony EXview HAD CCD II

- Optics: High resolution f/1.4 1" lens

- Resolution: 2750 x 2200 pixels

- High sensitivity: (QE 75\%), low smear

- Dark current: 0.002 e/pix/s @-10 ㄷ

- Cooling: Regulated Peltier $\Delta \mathrm{T}=-35^{\circ} \mathrm{C}$

- Digital Output: 16-bit 65536 levels

- Readout Speed: 6-12 MPixels/s

- Binning and Region-of-Interest

- External Trigger: GPIO synch.

Fig 3. High resolution imaging camera
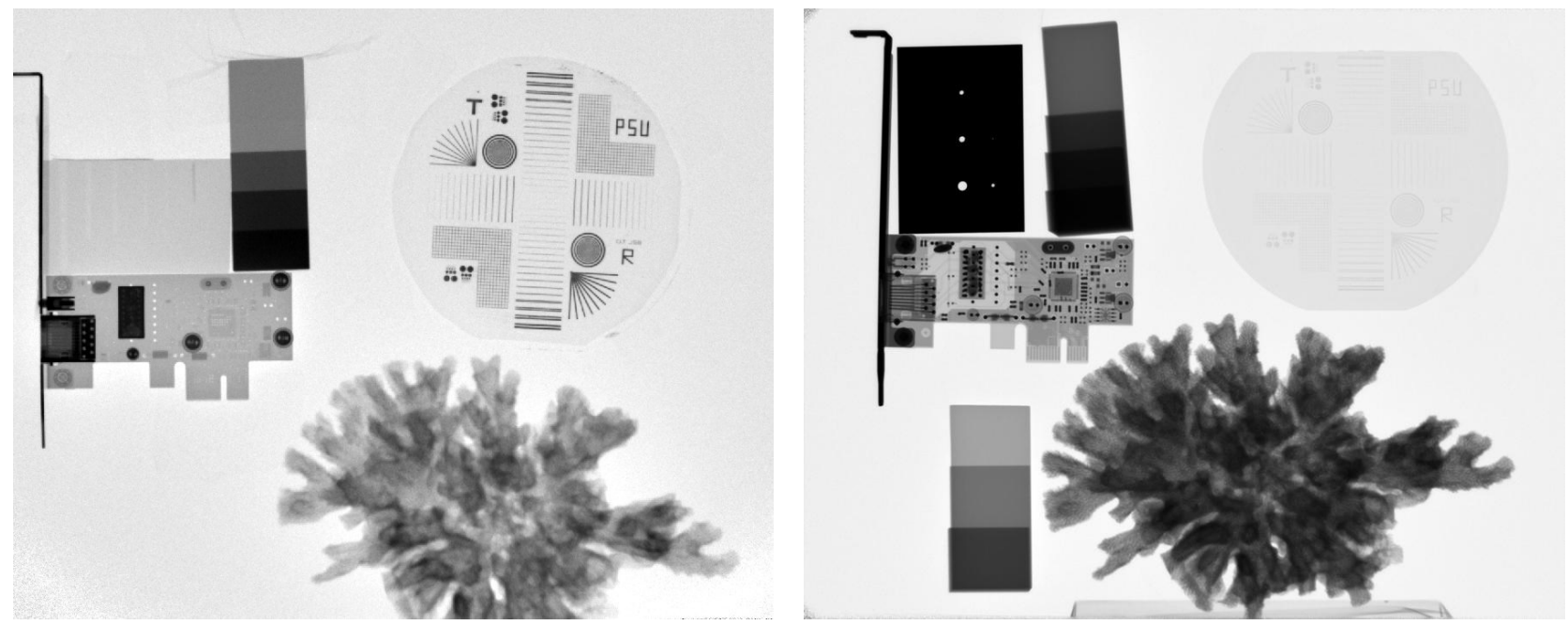

Fig 4a) Images on $100 \mathrm{~kW}$ Triga reactor (60s). Fig. 4b) on 60kV/3ma. x-ray source (15s)(R. Zboray PSU)

\section{Comparison of the Sony ICX694ALG 1-inch CCD \& Expensive CCD/CMOS Detectors}

The Well Depth is an important measure of the quality of a detector, since the Dynamic Range is the Well Depth divided by the Total Noise. So at first sight the Andor iKon has a big advantage (table 1 below). However, the Dark Current noise is exceptionally low for the Sony CCD, so the advantage is not so great in practice. In fact the well depth of the Sony CCD is equal to that of the PCO sCMOS chip, which is an excellent detector. Low dark current can only be achieved with the iKon using extreme cooling, which is certainly not an advantage, especially in an 
enclosure where air circulation would be limited. The external Sony camera achieves lower dark current with more modest Peltier-air cooling.

The iKon Read Noise is also higher unless very slow readout is used. The iKon publicity claims better numbers for individual parameters, but they cannot all be achieved simultaneously eg for readout at 1 frame/sec, the same as the Sony camera, the read noise is much higher at 31.5 ! The iKon is a good camera, but not significantly better for many users, especially considering that it is an order of magnitude more expensive, and also more expensive to maintain, repair and replace.

Table 1. Comparison of Sony ICX694ALG 1-inch CCD \& Expensive CCD/CMOS Detectors

\begin{tabular}{|c|c|c|c|c|}
\hline CCD & PCO.edge gold 4.2 & NOptics VS60 & NOptics 11002 & iKon L-936 \\
\hline Type & $\begin{array}{c}\text { Scientific sCMOS } \\
\text { CIS2020 }\end{array}$ & $\begin{array}{l}\text { Sony Interline } \\
\text { ICX694ALG }\end{array}$ & $\begin{array}{c}\text { "Kodak" Interline } \\
\text { KAl-11002 }\end{array}$ & $\begin{array}{c}\text { e2V Full Frame } \\
\text { CCD42-40 }\end{array}$ \\
\hline Resolution pixel & $2048 \times 2048$ & $2759 \times 2200$ & $4008 \times 2672$ & $2048 \times 2048$ \\
\hline Image diag. mm & $18.8(4 / 3 ")$ & $16(1 ")$ & $43.3(35 \mathrm{~mm})$ & $38(35 \mathrm{~mm})$ \\
\hline Image area $\mathrm{mm}$ & $13.3 \times 13.3$ & $12.53 \times 9.99$ & $37.25 \times 25.70$ & $27.6 \times 27.6$ \\
\hline Pixel size $\mu m^{\star}$ & $6.5 \times 6.5$ & $7.4 \times 7.4$ & $9.0 \times 9.0$ & $13.5 \times 13.5$ \\
\hline Quantum Effic* & $>70 \%$ & $75 \%$ & $50 \%$ & $90 \%$ \\
\hline Fullwell e- ** & $\sim 30,000$ & $\sim 30,000$ & $\sim 60,000$ & $\sim 100,000$ \\
\hline Read noise e- ** & 1 & 6 & 13 & 12 \\
\hline Dark c. e-/pix/s & $<0.02 @-30^{\circ} \mathrm{C}$ & $0.002 @-10^{\circ} \mathrm{C}$ & $0.03 @-20^{\circ} \mathrm{C}$ & $0.01 @-50$ C \\
\hline Peltier Cooling & $\Delta-30^{\circ} \mathrm{C}$ & $\Delta-35^{\circ} \mathrm{C}$ & $\Delta-38^{\circ} \mathrm{C}$ & $\Delta-80^{\circ} \mathrm{C}$ \\
\hline Read time $(\mathbf{s})^{\star \star \star}$ & 0.01 to 0.02 & 1 & 12 to 22 & 2 to 10 \\
\hline A/D Readout** & 16-bits & 16-bits & 16-bits & 16-bits \\
\hline Interface & USB 3.0 & USB 2.0 & USB 2.0 & USB 2.0 \\
\hline Relative Cost & 16 & 4 & 6 & 50 \\
\hline
\end{tabular}

\section{Very High Resolution Macro Neutron and X-ray Imaging}

Following the proposal of Kardjilov et al. [8] we have constructed an inexpensive 1:1 macro imaging camera designed to use the latest Gd2O2S:Tb high resolution neutron/x-ray scintillators from RC-TriTec. The photo shows our macro camera optics; without the CCD unit it is $290 \mathrm{~mm}$ high. With a large commercial $\mathrm{f}=100 \mathrm{~mm} \mathrm{f} / 2.8 \mathrm{~F}$-mount macro lens, the FOV is equal to the size of the CCD, and the optical resolution approaches the size of the pixels, though the resolution depends of course on the scintillator and collimation.

The camera is shown with a carbon fibre x-ray window, which unscrews to change the scintillator. The C-mount adapter can be unscrewed to take Nikon F-mount cameras up to 35mm full frame. Fine manual focus locking is provided by a thumbscrew mechanism; optional remote focusing can be provided by a motorised drive, controlled by a remote control box or computer.

A compact version using a shorter focal length lens gives larger FOVs with smaller CCDs. A 2x super resolution version can be made to order with an additional 50mm Rodenstock lens following Williams et al.[9].

The optical resolution of such a 1:1 neutron macroscope is comparable to the dimensions of

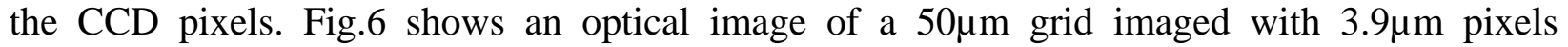
(checkered pattern) on our 1:1 macro imaging camera. The $25 \mu \mathrm{m}$ wires are clearly resolved to better than $10 \mu \mathrm{m}$ ( 2 pixels). 
The real resolution depends of course on the thickness of the scintillator, and for neutrons will be significantly less than the optical resolution. Although even higher optical magnification may be justified for $\mathrm{x}$-rays, there seems little point in that for neutrons; it is more important to maximise the signal/noise from the thin neutron scintillators [10].

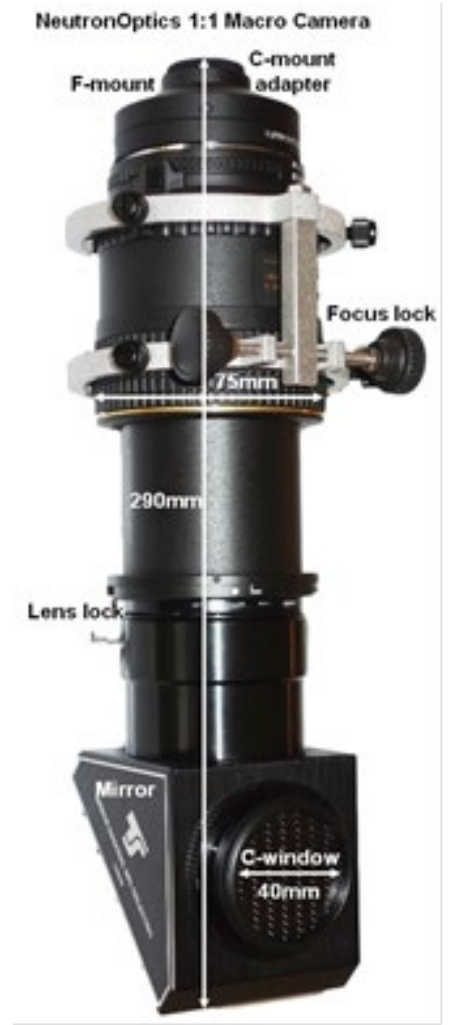

Fig 5. Neutron or X-ray Macro camera

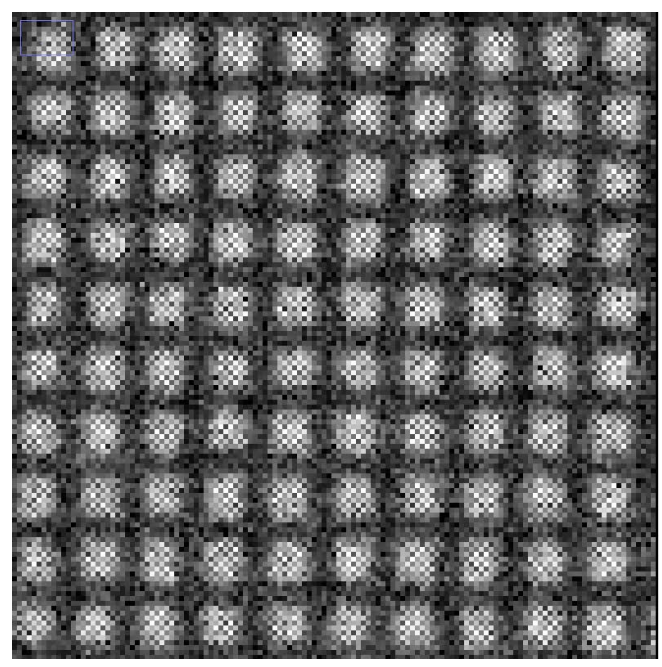

Fig.6 Optical image of $25 \mu \mathrm{m}$ wire grid

\section{Backscattering Laue Camera using a 1-inch Sony CCD}

CCD cameras have continued to be important for neutron and x-ray diffraction, and again simplification has been pursued, using only a single 1-inch CCD instead of multiple CCDs. Again the idea was to replace the $\mathrm{x}$-ray Laue camera, or image plate cameras used for crystal alignment in many labs. X-ray intensities are very low in Laue backscattering, so the camera (fig.7) has been optimised to be as close to the source as possible, with the 1-inch CCD permitting a relatively large $120 \times 100 \mathrm{~mm}$ window and collection times of 2-3 minutes on normal x-ray generators. (Si and Sm2Fe17 micro-crystal mages figs.8 below courtesy of Dean Hudek (Brown University) and Wolfgang Donner \& Léopold Diop (TU Darmstad). 


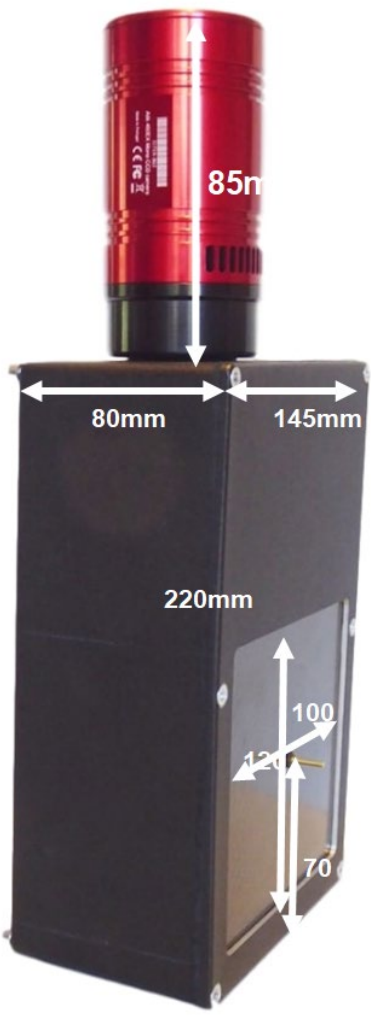

Fig.7 Laue x-ray camera

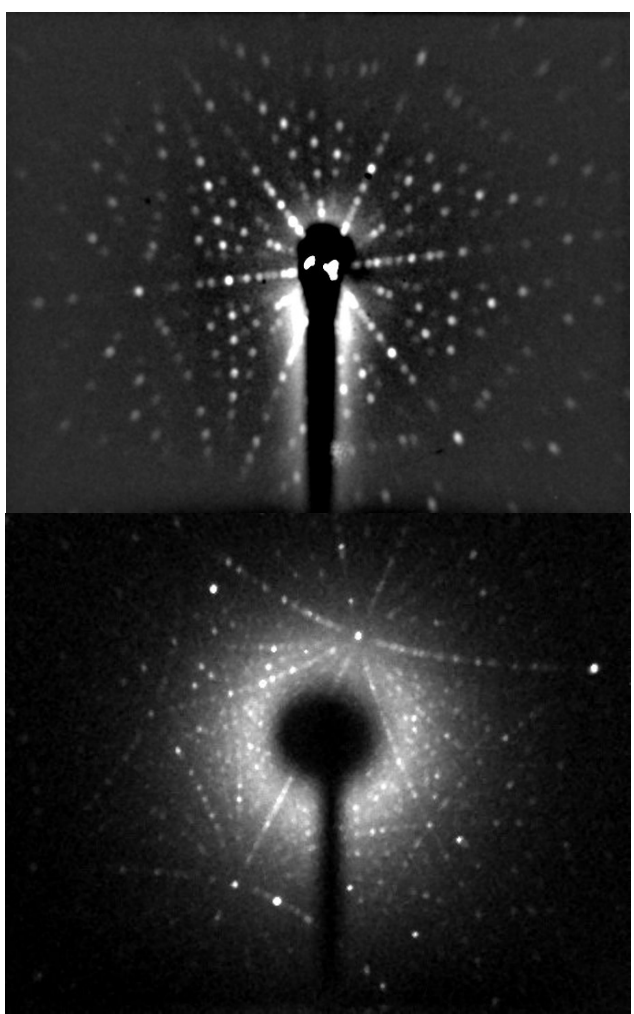

Fig.8a Si (top) and Sm2Fe17 (bottom) imges

\section{Conclusion and Summary}

We have shown that the largest Sony CCD, the 1" ICX694ALG is a good choice for a wide variety of neutron and x-ray imaging cameras. It is much less expensive to purchase and maintain than specialised cameras used in big laboratories, yet competes well with such cameras, even on low flux sources. We have given examples of an efficient 250x200mm camera with an optical resolution of $90 \mu \mathrm{m}$, a 1:1 macro camera with an optical resolution of better than $10 \mu \mathrm{m}$, and a single-CCD Laue camera that can provide rapid alignment of single crystals on an ordinary $\mathrm{X}$-ray generator.

\section{References}

[1] Arndt, U.W. \& Ambrose, B.K. (1968) in Cambridge (UK) "An Image Intensifier - Television System for the Direct Recording of X-ray Diffraction Patterns", IEEE. Trans. Nucl. Sci. NS-15, 92-94. https://doi.org/10.1109/TNS.1968.4324920

[2] Brunner, J., A. Hillenbach, E. Lehmann, and B. Schillinger. (2002) "Dynamic neutron radiography of a combustion engine." In Proc. 7th World Conf. on Neutron Radiography, Rome, p. 439.

[3] Tengattini, A., D. Atkins, B. Giroud, E. Andò, J. Beaucour, and G. Viggiani.(2017) "NeXTGrenoble, a novel facility for Neutron and X-ray Tomography in Grenoble." In Proc. 3rd International Conference on Tomography of Materials and Structures (Lund, Sweden, 26-30 June 2017). https://next-grenoble.fr/

[4] A. Hewat \& P. Falus (2007) ILL Annual Report pp. 85-86

"An_Inexpensive_CCD_Neutron_Alignment_Camera" 
[5] Ouladdiaf, B., Archer, J., McIntyre, G. J., Hewat, A. W., Brau, D. \& York, S. (2006). "OrientExpress: A new system for Laue neutron diffraction" Physica B, 385-386, 1052-1055. https://doi.org/10.1016/j.physb.2006.05.337

[6] A.W. Hewat, B. Ouladdiaf, G.J.McIntyre, M-H.Lemee-Cailleau, D. Brau, S. York (2006) "CYCLOPS, A proposed high flux CCD neutron diffractometer" ILL Millennium Programme Symposium 2006

[7] Bachir Ouladdiaf, John Archer, John R. Allibon, Philippe Decarpentrie, Marie-Helene Lemee-Cailleau, Juan Rodriguez-Carvajal, Alan W. Hewat, Scott York, Daniel Brau and Garry J. McIntyre (2011) "CYCLOPS - a reciprocal-space explorer based on CCD neutron detectors" J. Appl. Cryst. 44, 392-397. https://doi.org/10.1107/S0021889811006765

[8] Kardjilov, N., M. Dawson, A. Hilger, I. Manke, M. Strobl, D. Penumadu, F. H. Kim, F. Garcia-Moreno, and J. Banhart. "A highly adaptive detector system for high resolution neutron imaging." Nuclear Instruments and Methods in Physics Research Section A: Accelerators, Spectrometers, Detectors and Associated Equipment 651, no. 1 (2011): 95-99. https://doi.org/10.1016/j.nima.2011.02.084

[9] Williams, S. H., A. Hilger, N. Kardjilov, I. Manke, M. Strobl, P. A. Douissard, T. Martin, Heinrich Riesemeier, and J. Banhart. "Detection system for microimaging with neutrons." Journal of Instrumentation 7, no. 02 (2012): P02014. https://doi.org/10.1088/17480221/7/02/P02014

[10] Walfort, B., Grünzweig, Ch., Trtik, P., Morgano, M. and Strobl, M. (2018)

"Novel scintillation screen with significantly improved radiation hardness and very high light output" WCNR-11 Sydney proceedings. 